\title{
BMJ Open Quality Reducing time to treatment of bacterial sexually transmitted infections in an HIV cohort
}

\author{
Richard Kennedy, Andrew J Winter
}

To cite: Kennedy R, Winter AJ. Reducing time to treatment of bacterial sexually transmitted infections in an HIV cohort. BMJ Open Quality 2020;9:e000603. doi:10.1136/ bmjoq-2018-000603

Received 13 December 2018 Revised 24 February 2020 Accepted 23 March 2020
Check for updates

(C) Author(s) (or their employer(s)) 2020. Re-use permitted under CC BY-NC. No commercial re-use. See rights and permissions. Published by BMJ.

Brownlee Centre, Gartnavel General Hospital, Glasgow, UK

Correspondence to Dr Richard Kennedy; richardkennedy3@nhs.net

\section{ABSTRACT}

Sexually transmitted infections (STIs) are known to increase the risk of transmission of HIV and care of sexual health needs should form part of routine HIV care. Delayed treatment of STIs can lead to complications and avoidable onward transmission. Management of acute STIs in UK specialist sexual health services usually involves a multidisciplinary approach to ensure patient recall, antimicrobial treatment and partner notification. While this works well in dedicated sexual health clinics, we found this was less optimal in our hospital-based HIV care unit. We describe a quality improvement project to improve interdisciplinary pathways by using electronic shared worklists that reduced time to treatment for chlamydia and gonorrhoea infections. Use of electronic shared worklists could be applied to other settings where rapid treatment is required or has transmission implications.

\section{PROBLEM}

Management of positive sexually transmitted infection (STI) results at our large urban National Health Service (NHS) HIV unit have, prior to now, relied on paper copies of results reaching staff. In an age where these results are available much quicker electronically through online patient management systems, this was seen as an unnecessary delay in the management of positive STI results, with implications for both the onward transmission of bacterial STIs and potentially HIV infection. This quality improvement project was aimed at the implementation of electronic worklists (with shared HIV sexual health advisor (SHA) and HIV clinician use) to reduce the average monthly time taken to treatment of a confirmed STI in our HIV clinic's cohort within a 12-month period. This would be measured by the percentage of months surpassing national average time to treatment, from a baseline of $50 \%$ to an aim of more than $90 \%$. Additional benefits of this project, although not formally measured, were to improve communication between disciplines working within the HIV unit and clearer documentation of management of STIs in the patients' notes.

\section{BACKGROUND}

Concurrent STIs are known to increase the risk of HIV transmission in both male and female persons living with HIV (PLWH). ${ }^{1-3}$ As such, STI screening is now recommended as part of routine HIV care. ${ }^{45}$ Improvements in management of STIs in PLWH has been hypothesised to impact on HIV prevalence, ${ }^{6}$ and rapid treatment of STIs has also been suggested as an important factor in this population. ${ }^{7}$ It is estimated that even in those symptomatic of STIs, approximately $50 \%$ of people will continue to have sex while they await treatment (4\% with more than one partner), ${ }^{8}$ with higher rates expected in those who are asymptomatic. Prompt treatment of STIs is therefore of importance to reduce onward transmission of STIs. The national average for time to treatment by a sexual health service in the UK using booked appointments is 10 days. ${ }^{8}$

Our local HIV cohort of 1825 patients is jointly managed by infectious disease (ID) and genitourinary medicine (GUM); however, both teams operate NHS HIV care from one site, meaning HIV SHAs, who manage these infections and counsel patients regarding safer sex, can be centralised to a single base. Preimplementation, outstanding tests for routine sexual health screens (SHS) tests were added to a paper diary with a text sent to patients thereafter when results were available. Results for routine SHS tests taken by doctors or clinical nurse specialists involved the patient's details being given to a SHA ad hoc and added to the paper diary for the above process to take place-for example, patient details given to SHA in clinic, patient details left on SHA desk or patients discussed at multidisciplinary team meeting or in passing. Results for chalmydia, gonorrhoea and syphilis reached the HIV SHAs in hardcopy paper format, who then managed these results appropriately. Depending on the result, this may have been sending the patient a text to inform them that the results were negative, 


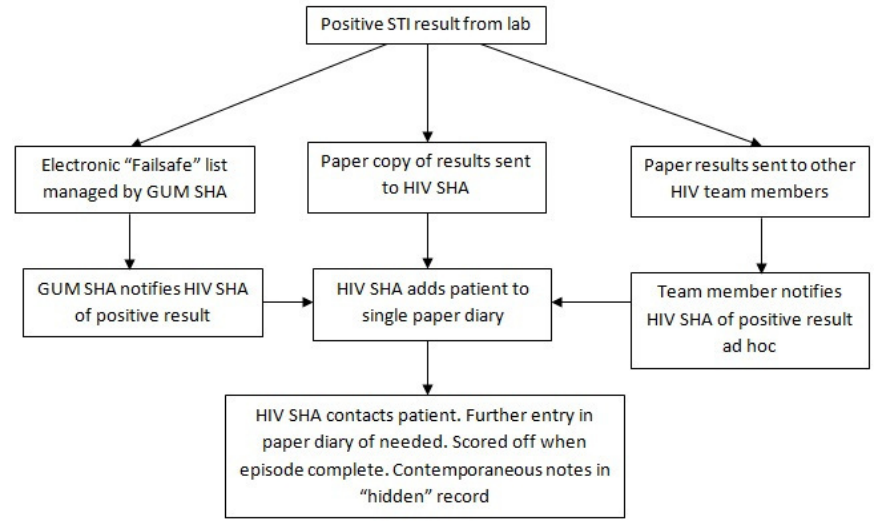

Figure 1 Results management pathway prior to intervention. GUM, genitourinary medicine; SHA, sexual health advisor; STI, sexually transmitted infection.

or making attempts to recall the patient for treatment if results were positive. We operate a flexible booked appointment service for patients attending SHAs for both treatment and screening. Should this process have missed any positive results, there exists an automated 'Fail Safe' mechanism via a separate SHA office in the local GUM clinic. Here, the GUM SHA team are directly notified electronically of any positive SHS results identified by the local laboratories. HIV SHAs are then contacted by the GUM SHA team to confirm that the infection has been managed. However, since electronic results tended to be available sooner than paper versions, the Fail Safe system was often the first notification the HIV SHA office had of positive results and therefore not fulfilling its designed purpose as a back-up facility. For positive results, management outcomes were recorded in a 'hidden' version of our electronic notes platform and stored securely in our local electronic patient management system, making finding this information lengthy and sometimes overlooked. Figure 1 shows the results management pathway prior to intervention.

\section{MEASUREMENT}

As a measure for how effective changes to this process were, we looked at time to treatment of bacterial STIs (chlamydia and gonorrhoea). Bacterial STIs were retrospectively identified from positive results highlighted through the 'Fail Safe' system that were then cross-referenced with patient identifiers of those attending our HIV clinic. A clinical notes review was then conducted to identify documented date of treatment. Most individuals sought treatment at either our HIV care site itself or at our local GUM clinic, and notes for both sites were reviewed where relevant. The number of working days (Monday-Friday) between the date the sample was taken and date of treatment was then calculated. Syphilis results were not included in measurements as a large number of positive syphilis serology results are used for monitoring and follow-up of patients and usually do not require any particular further treatment. Treatment of other bacterial STIs was felt to be a suitable proxy marker for all treatment pathways. Multiple infections from the same testing day in the same individual (ie, chlamydia and gonorrhoea infection) were counted as a single infective episode, as were multiple infected sites from one episode (eg, throat and rectal chlamydia). Similarly, multiple tests within the same episode (eg, repeat samples taken on the day the patient was seen for treatment) were counted as a single infective episode. Those who did not attend for treatment despite recall were excluded from final analysis, as were those who sought treatment out-with our health board as we could not verify date of treatment in these individuals.

\section{DESIGN}

On reviewing areas where time to treatment of positive STI results could be reduced in our HIV cohort, in a climate where both our local HIV cohort and the incidence of bacterial STIs continues to climb, ${ }^{9}$ the results management process was identified as a potential delay. Results are available electronically through our local electronic patient management system, and it was hypothesised that an editable electronic worklist of outstanding results would allow more rapid and streamlined access to these results with subsequent reduction in time to treatment of positive results. The preimplementation process was also felt to be less robust than a potential electronic results process, as paper results can easily be lost or delivered to the wrong address. Furthermore, an electronic worklist is in line with our current local move towards paper-light electronic records and electronic handling of results. Electronic rather than hardcopy lists also allow remote access to the HIV SHA service when medical staff are off-site. Within our health board, electronic results appear under a named staff member, usually based on the named person in charge of the clinic where the sample was taken. This may be a senior medical team member or a senior SHA team member, and one team may not necessarily check the other's results. With multiple staff groups involved in the obtaining and management of these tests, there can therefore be fragmentation of care. For example, if a medical staff member identifies a positive STI result that the SHA team have not seen, they need to alert the SHA team so that the patient can be contacted and recalled for treatment. Similarly, if the SHA team identify a result that requires a medical opinion or a prescription, they must find a medical staff member to do this. It was hoped that having these worklists and an accountable person for managing them daily could act as an accessible and robust communication tool between staff disciplines. In summary, it was hoped an exclusively electronic system for handling and managing STI results compared with paper results or the primary use of the 'Fail Safe' system would cut down on missing results, lengthy chasing down of colleagues, requests being sent to off-duty staff, duplication of work and ultimately delays to time of treatment. A group of individuals were involved in creating the protocols for this quality improvement process. This consisted of several medical staff (including our health board's joint clinical lead for e-Health) and SHA 


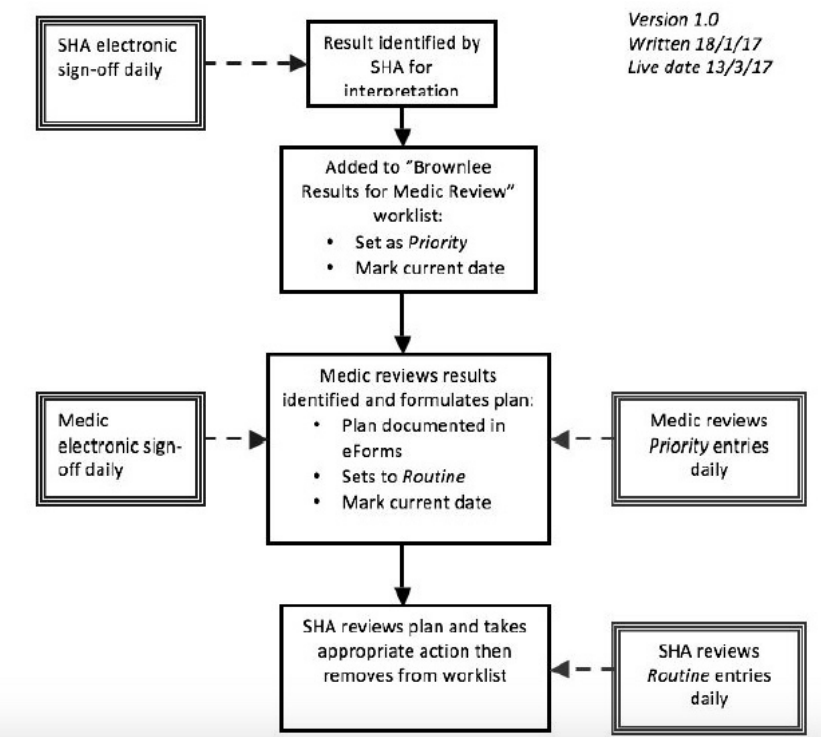

Figure 2 Worklist 1 flow chart: results requiring a GUM physician review. GUM, genitourinary medicine; SHA, sexual health advisor.

staff (including the local SHA line manager and president of Society of Sexual Health Advisors).

Documentation of management of positive results was to be entered into the generic patient notes, rather than a hidden GUM note. It is worth noting that only those working within the HIV service have access to these generic notes. However, if both HIV and sexual care was being documented in the same place, it would avoid any missed notes or duplication of work. This is of further importance due to the imminent update of our health board's electronic notes system that will mean the use of a single form.

Ethical considerations were the impact on patient experience, staff experience and impact on wasted resource. It was not anticipated that this intervention would result in any patient experiencing a negative impact on their care, as how patients were contacted and treated would not be affected, only the time to treatment. It is also noted that the introduction of new systems brings with it the possibility of new system errors. One potential new weakness identified in this system is that once patients are deleted from the electronic worklist, there is no way to retrieve this entry, and it must be manually added again. This would be monitored by measuring how many infections were not treated. Staff apprehension regarding a new system was taken into consideration, and staff members were fully supported throughout the project. Finally, this process was aimed to reduce the use of paper, as is encouraged throughout our health board.

\section{STRATEGY}

The worklists were created through our health board's electronic notes platform (Orion's Clinical Portal). This software was already available to all staff in the department, as well as a worklist administrator, and therefore no additional costs were required for this. The worklists allow the patients to be added directly to the worklist, while staff are accessing the patients notes (eg, during clinic or when entering notes about a patient), and vice versa the patients results and contact details can be accessed directly from the worklist. Worklists can be accessed by anyone who is added to the user group by the worklist administrator, who works within our team and was involved in the project strategy group. Each entry on the worklist has a priority ranking ('priority' or 'routine'), a date to be actioned by and a notes section where specific instructions or further details can be easily seen. Clear roles and responsibilities in managing results and use of the worklists for both medical and SHA teams were set out in a protocol and relevant staff briefed on how worklists should be used. It was decided to pilot utility of the worklists within the GUM medical team initially and, if successful, to expand access to the worklists to also include the ID medical team. Staff members were informally consulted throughout the duration of the implementation process to ensure they were satisfied with process and discuss any issues that may have arisen, with a feedback session between the project leads and the HIV SHAs 4 months after the introduction of the worklists. After group consultation, the first version of our protocol was produced, and worklists were created. It was proposed between the group described above that two worklists would be required. The first worklist was for SHAs to request medical staff review of sexual health related results that are out-with their proficiencies, and this went live in March 2017. This was anticipated to largely be review of syphilis serology. Interpretation of syphilis serology can be difficult, particularly in assessing response to treatment and reinfection ${ }^{10}$ and may require GUM physician review. The worklist could also be used for other STI results that need further interpretation such as inhibited or indeterminate nucleic acid amplification test samples, which are the gold standard screening tests for both chlamydia and gonorrhoea, ${ }^{11} 12$ or requesting prescriptions for treatment. Figure 2 shows the new process and flow chart included in the worklist protocol, which staff could refer to.

The second worklist was created to allow SHAs to keep an electronic record of outstanding sexual health related tests or results that need chased or acted on. This may be a SHS that is taken by medical staff in clinic on a patient who wishes contacted with results when they are available, a positive result that has been electronically allocated to medical staff requiring treatment or a sample that needs repeated. Both medical and SHA team members can access this worklist and add or remove patients as indicated. This worklist also went live in March 2017. The date function within the worklist was used for tests that have been performed and results need chased at a future date, and the priority function was used to alert SHAs of positive results that needed treated. Figure 3 shows the new process and flow chart from the worklist protocol that was used as a reference guide for staff. 


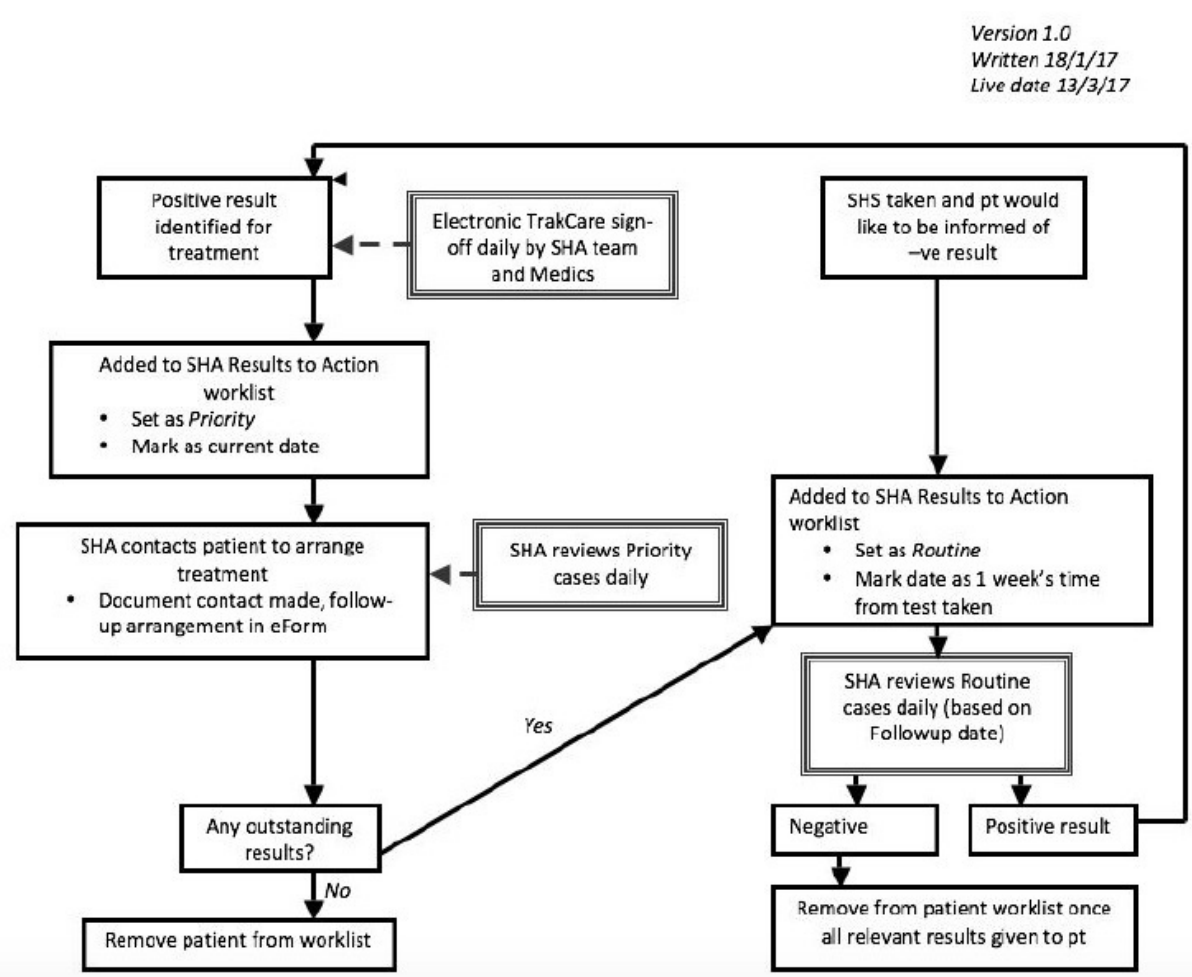

Figure 3 Worklist 2 flow chart: outstanding results for HIV SHA to act on. SHA, sexual health advisor; SHS, sexual health screens.

\section{Patient and public involvement}

Patient groups were not specifically involved in the set-up of this quality improvement protocol development.

\section{RESULTS}

All chlamydia and gonorrhoea positive samples received from our clinic's patients with HIV from May 2016 to January 2018 (inclusive) were included in analysis $(\mathrm{n}=138)$. As detailed above, those with multiple infections $(n=8)$ or multiple infection sites $(n=2)$, or those with multiple tests for the same infection in the same treatment episode $(n=9)$ were counted as single infective episodes. Those where treatment was not given or treatment date was not verifiable from clinical notes (such as accessing treatment at another facility) were excluded from analysis $(n=6)$. Patients under the care of the ID team within our cohort were excluded from the postimplementation analysis $(n=15)$ as the worklists were piloted among the GUM clinicians only, as detailed above.

Figure 4 shows a summary of the results. Monthly averages for time to treatment were taken. The average monthly time to treatment prior to implementation was over the national average in $50 \%$ of months studied. Following intervention, this was reduced to $9 \%$ of months. Following 1 month where time to treatment was more than the national average, a further session was held with the HIV SHA team to review the process and address any questions or concerns they had. Beyond this, monthly averages were maintained below the national average.
One hundred per cent of infective episodes were effectively treated or had recall attempted at least twice.

\section{Lessons and limitations}

This project aimed to reduce time to treatment of positive STI results, and this effect was seen and sustained following our interventions. This approach to STI results management was novel within this HIV clinic. It removes the need for a single hardcopy diary for management of positive STI results, thus addressing confidentiality issues and improving staff access by allowing multiple members of the team to use the facility from any site within the health board with computer access. Furthermore, this new system reduces paper use as all results can now be accessed directly through the local electronic patient management system. In terms of reduction of paper, we have anecdotally grossly reduced the number of paper results that come through our HIV department. This new way of working has allowed us to align STI result pathways to that of other lab results in being exclusively electronically managed and has not been formally measured. Limitations of this analysis include the small sample size (largely due to the recentness of implementation), and a larger sample size may have produced a more significant effect. A further limitation to this project was the implementation of changes at a single time point rather than in a series. It was felt that changing all paper to electronic worklists on a single date would be easier for staff to adopt than staggering these. Effect on time to STI treatment was also limited due to human factors in treating infections, 


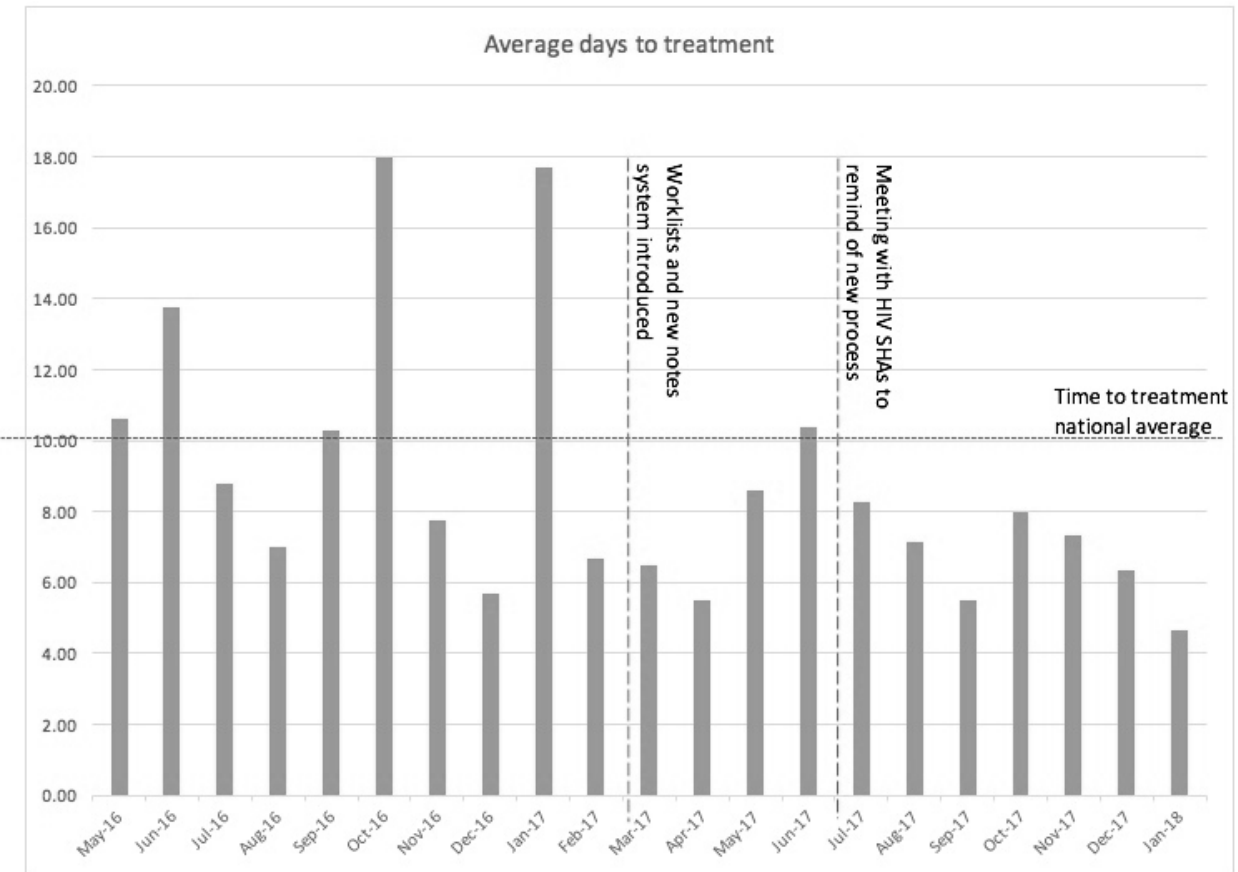

Figure 4 Monthly average of time to treatment (working days) and implementation timeline. SHAs, sexual health advisors.

both in terms of clients and providers. There is an obvious limit to how much time to treatment can be reduced due to the time it takes for the laboratory to receive and process the sample. Additionally, despite even the promptest recall for a positive result, many patients could not attend for several days, limiting the extent to which time to treatment can be reduced, despite potentially reducing the time to informing a patient of a positive result. It is worth noting that time to informing patients was not always readily available from notes so it was therefore not used as a measure for improvement in this project. Reassuringly, no infections were missed in our analysis, and the Fail Safe system will continue to identify any patients that may fall victim to such system errors, suggesting the risk of names being inadvertently deleted off worklists is unlikely to be clinically significant. Another limitation to the worklists themselves are that a patient cannot appear on the same worklist more than once, should they have more than one issue needing follow-up. This means follow-up dates need to be amended as each issue is resolved.

It is acknowledged that other factors may have contributed to the effect seen, and reduced time to treatment in the postimplementation group may not have been solely due to electronic management of results. Other possible contributors may include staffing and availability of independent prescribers (although of note the site went from three SHAs to two, and two independent SHA prescribers to one in the studied time frame), or more rapid lab turnaround of results. It was initially hoped to measure the latter in addition to time to treatment, but due to limitations on how the results are reported on the electronic patient management system, this was found during data collection to be inaccurate and was therefore not used in final analysis. We therefore feel the most likely contributor to this time reduction is our QI interventions.

During the process of this implementation, several other observations were made and lessons learnt. First, the use of the worklists was designed to be limited to a very particular remit. Despite this, SHA staff felt they required an electronic list of planned follow-up of patients (such as test of cure dates for gonorrhoea ${ }^{11}$ or follow-up of syphilis serology ${ }^{1013}$ ) and often used the second worklist detailed above to facilitate this. One suggestion has been to create a shared email diary where these outstanding tests can be documented instead, and this is currently being investigated. Finally, this project involved a significant degree of staff adaptation, as well as ongoing staff training, support and feedback. Informal feedback from both medical and SHA staff is that this has improved the results management system.

\section{CONCLUSION}

One year following the implementation of an electronic worklist for management of positive bacterial STI results within an HIV clinic setting, a reduced time to treatment of chlamydia and gonorrhoea in this cohort was observed, as set out in the project's aim. Given that we know people will continue to have sexual intercourse even when symptomatic, ${ }^{8}$ even modest reductions in number of days to treatment of STIs can potentially reduce the number of partner exposures. It is estimated that the average delay to attending for treatment due to patient factors is 7 days, ${ }^{8}$ so improvement beyond this is unlikely to be realistic. Provider delay is also estimated at, on average, 7 days for those using a mixture of walk-in and booked appointments. When we compare to booked appointment services 
(such as ours), we now perform better than the estimated average provider delay of 10 days, ${ }^{8}$ compared with being above this estimated average prior to implementation. In addition to reduced time to treatment, some further general positive trends in patient care were noted. First, all positive STI results were either treated or appropriately recalled (at least twice). Second, following implementation, the management of positive STI results was much clearer with use of a visible (to preapproved users) electronic note and discontinued use of the 'hidden' GUM notes.

This quality improvement project demonstrated that the introduction of online, accessible, electronic worklists for multidisciplinary results management is feasible and is likely to contribute to reduced time to appropriate management of abnormal results.

Acknowledgements With thanks to the following for their contribution to this work: Ms Revathy Raajaravi, Mr Samuel King, sexual health advisor (SHA) team at Brownlee Services and GUM medical staff for their adaptation to this new approach.

Contributors With thanks to the following for their contribution to this work: Ms Revathy Raajaravi and Mr Samuel King for data collation. The Sexual Health Advisor team at Brownlee Services and all GUM medical staff in NHS GG\&C for their adaptation to this new approach. RK and AJW planned the study. RK conducted study measurements. RK and AJW are responsible for the content of the report. RK submitted the study.

Funding The authors have not declared a specific grant for this research from any funding agency in the public, commercial or not-for-profit sectors.

Competing interests None declared.

Patient and public involvement Patients and/or the public were not involved in the design, or conduct, or reporting, or dissemination plans of this research.

Patient consent for publication Not required.

Provenance and peer review Not commissioned; externally peer reviewed.

Data availability statement All data relevant to the study are included in the article.

Open access This is an open access article distributed in accordance with the Creative Commons Attribution Non Commercial (CC BY-NC 4.0) license, which permits others to distribute, remix, adapt, build upon this work non-commercially, and license their derivative works on different terms, provided the original work is properly cited, appropriate credit is given, any changes made indicated, and the use is non-commercial. See: http://creativecommons.org/licenses/by-nc/4.0/.

\section{REFERENCES}

1 Sadiq ST, Taylor S, Kaye S, et al. The effects of antiretroviral therapy on HIV-1 RNA loads in seminal plasma in HIV-positive patients with and without urethritis. AIDS 2002;16:219-25.

2 Gitau RW, Graham SM, Masese LN, et al. Effect of acquisition and treatment of cervical infections on HIV-1 shedding in women on antiretroviral therapy. AIDS 2010;24:2733-7.

3 Laga M, Manoka A, Kivuvu M, et al. Non-Ulcerative sexually transmitted diseases as risk factors for HIV-1 transmission in women: results from a cohort study. AIDS 1993;7:95-102.

4 Fakoya A, Lamba H, Mackie N, et al. British HIV association, $\mathrm{BASHH}$ and FSRH guidelines for the management of the sexual and reproductive health of people living with HIV infection 2008. HIV Med 2008;9:681-720.

5 Nandwani R. 2006 United Kingdom national guideline on the sexual health of people with HIV: sexually transmitted infections. Int J STD AIDS 2006;17:594-606.

6 Hayes R, Watson-Jones D, Celum C, et al. Treatment of sexually transmitted infections for HIV prevention: end of the road or new beginning? AIDS 2010;24 Suppl 4:S15-26.

7 Steen R, Wi TE, Kamali A, et al. Control of sexually transmitted infections and prevention of HIV transmission: mending a fractured paradigm. Bull World Health Organ 2009;87:858-65.

8 Mercer $\mathrm{CH}$, Sutcliffe L, Johnson AM, et al. How much do delayed healthcare seeking, delayed care provision, and diversion from primary care contribute to the transmission of STIs? Sex Transm Infect 2007;83:400-5.

9 Health Protection Scotland and Glasgow Caledonian University. Blood borne viruses and sexually transmitted infections: Scotland 2017. Glasgow: Health Protection Scotland, 2017.

10 Ratnam S. The laboratory diagnosis of syphilis. Can J Infect Dis Med Microbiol 2005;16:45-51.

11 Bignell C, Fitzgerald M, Group GD. British association for sexual health and HIV UK. UK national guideline for the management of gonorrhoea in adults, 2011. Int J STD AIDS 2011;22:541-7.

12 Nwokolo NC, Dragovic B, Patel S, et al. 2015 UK national guideline for the management of infection with Chlamydia trachomatis. Int $J$ STD AIDS 2016;27:251-67.

13 Kingston M, French P, Higgins S, et al. Uk national guidelines on the management of syphilis 2015. Int J STD AIDS 2016;27:421-46. 